\title{
Polarimetric multiphoton microscopy for the analysis of ocular structures
}

\section{Microscopía multifotónica polarimétrica para el análisis de estructuras oculares}

\author{
Francisco J. Ávila*s, Oscar Del Barco, Juan M. Buenos \\ Laboratorio de Óptica, Instituto Universitario de Investigación en Óptica y Nanofísica, \\ Universidad de Murcia, Campus de Espinardo, 30100 Murcia, España \\ (*) E-mail: avila@unizar.es $\quad$ S: miembro de SEDOPTICA \\ Received: 10/10/2018 Accepted: 01/02/2019 \\ DOI: 10.7149/OPA.52.1.51013
}

\begin{abstract}
:
This review article summarizes the main contributions of the PhD Thesis titled "Polarization and multiphoton microsocopy for the analysis of ocular structures" carried out at the Laboratorio de Óptica of the University of Murcia. This work presents mathematical methods developed to both analyze and classify the external or micrometric scale of type I collagen-based ocular tissues. On the one hand, a theoretical model has been developed to explore the polarization dependence of second harmonic generation signal. This allowed obtaining an analytical expression to calculate the internal or molecular collagen organization. On the other hand, an experimental relationship between external and internal collagen organization has been stablished. Finally, the Stokes-Mueller formalism has been applied to compute the polarization properties of type-I fibrillar collagen and to improve the visualization of local structures. These methods have been proposed as a differential clinical tool for the diagnosis of corneal keratoconus.
\end{abstract}

Keywords: Non-linear microscopy, second harmonic generation, polarization, collagen.

\section{RESUMEN:}

Este artículo de revisión resume las principales aportaciones de la Tesis Doctoral "Polarización y microscopía multifotónica para el análisis de estructuras oculares" realizada en el Laboratorio de Óptica de la Universidad de Murcia. En ella se han desarrollado métodos matemáticos para analizar y clasificar la estructura externa o micrométrica de los tejidos oculares formados por colágeno tipo I. Por otra parte, se ha desarrollado un modelo teórico para analizar la dependencia con la polarización de la señal de segundo armónico y obtener una expresión analítica para el cálculo de la organización interna o molecular del colágeno. Así mismo se ha establecido la relación experimental entre orden externo e interno usando un microscopio multifotónico polarimétrico. Finalmente se ha aplicado el formalismo Stokes-Mueller para el cálculo de las propiedades de polarización del colágeno así como la mejora de visualización de estructuras locales. Los métodos desarrollados se han propuesto como herramienta diferencial de diagnóstico en la enfermedad ocular conocida como queratocono.

Palabras clave: Microscopía no lineal, generación de segundo armónico, polarización, colágeno.

\section{REFERENCIAS Y ENLACES}

[1] L. Klein, J. Chandrarajan, "Collagen degradation in rat skin but not in intestine during rapid growtheffect on collagen type-1 and type-2 from skin,” Proc. Nat. Ac. Sci. USA, 74(4), 1436-1439 (1977).

[2] Y. Komai, T. Ushiki, "The 3-dimensional organization of collagen fibrils in the human cornea and sclera," Invest. Ophthalmol. Vis. Sci. 32(8), 2244-2258 (1991).

[3] R. Seeley, T. Stephens, P. Tate, "Anatomy and Physiology," McGraw-Hill (2003). 
[4] S. Roth, I. Freund, "Second harmonic generation in collagen," J. Chem. Phys. 70(4), 1637-1643 (1979).

[5] M. M. Jhons, V. Kolachala, E. Berg, S. Muller, F.X. Creighton, R.C. Branski, "Radiation Fibrosis of the Vocal Fold: from man to mouse," Laryngoscope 122(5), S107-S125 (2012).

[6] B. F. Hochheimer, "Second harmonic light generation in the rabbit cornea," Appl. Opt. 21(8), 15161518 (1982).

[7] M. Han, G. Giese, J. Bille, "Second harmonic generation imaging of collagen fibrils in cornea and sclera," Opt. Express 13(15), 5791-5797 (2005).

[8] N. Morishige, A. J. Wahlert, M. C. Kenney, D. J. Brown, K. Kawamoto, T. Chikama, T. Nishida, J. V. Jester, "Second-harmonic imaging microscopy of normal human and keratoconus cornea," Invest. Ophthalmol. Vis. Sci. 48(3), 1087-1094 (2007).

[9] J. M. Bueno, E. J. Gualda, A. Giakoumaki, P. Pérez-Merino, S. Marcos, P. Artal, "Multiphoton microscopy of ex-vivo corneas after collagen crosslinking," Invest. Ophthalmol. Vis. Sci. 52(8), 5325-5331 (2011).

[10] Y. Chang, C. Chen, J. Chen, Y. Jin, X. Deng, "Theoretical simulation study of linearly polarized light on microscopic second-harmonic generation in collagen type I," J. Biom. Opt. 14(4), 044016 (2009).

[11] O. del Barco, J. M. Bueno, "Second harmonic generation signal in collagen fibers: role of polarization, numerical aperture, and wavelength," J. Biom. Opt. 17(4), 045005 (2012).

[12] I. Gusachenko, G. Latour, M.C. Schanne-Klein, "Polarization-resolved Second Harmonic microscopy in anisotropic thick tissues," Opt. Express 18(18), 19339-19352 (2010).

[13] I. J. Su, W. L. Chen, Y. F. Chen, C.Y. Dong, "Determination of Collagen Nanostructure from Second-Order Susceptibility Tensor Analysis," Biophysical J. 100(8), 2053-2062 (2011).

[14] F. Ávila, J. Bueno, "Analysis and quantification of collagen organization with the structure tensor in second harmonic microscopy images of ocular tissues," Appl. Optics, 54(33), 9848-9854 (2015).

[15] F. Ávila, O. Barco, J. Bueno, "Polarization dependence of collagen-aligned tissues imaged with second harmonic generation microscopy," J. Biom. Opt. 20(8), 086001 (2015).

[16] F. Ávila, O. Barco, J. Bueno, "Polarization response of second-harmonic images for different collagen spatial distributions," J. Biom. Opt. 21(6), 066015 (2016).

[17] F. Avila, O. Barco, J. Bueno, "Quantifying external and internal collagen organization from Stokesvector-based second harmonic generation imaging polarimetry," J. Opt. 19(10), 105301 (2017).

[18] J. M. Bueno, E. J. Gualda, P. Artal, "Adaptive optics multiphoton microscopy to study ex vivo ocular tissues," J. Biomed. Opt. 15 (6), 066004 (2010).

[19] A. Ambirajan, D. C. Look, "Optimum angles for a polarimeter: part I," Opt. Eng. 34(6), 1651-1655 (1995).

[20] J. M. Bueno, J. J. Hunter, C. J. Cookson, M. L. Kisilak, M. C. Campbell, "Improved scanning laser fundus imaging using polarimetry," J. Opt. Soc. Am. A 24(5), 1337-48 (2007).

[21] B. Jahne, "Spatio-temporal image processing: theory and scientific aplications," Springer, Berlin (1993).

[22] C. Odin, Y. Le Grand, A. Renault, L. Gailhouste, G. Baffet, "Orientation fields of nonlinear biological fibrils by second harmonic generation microscopy," J. Microsc. 229(1), 32-38 (2008).

[23] R. Hristu, S.G. Stanciu, D.E. Tranca, G.A. Stanciu, "Improved quantification of collagen anisotropy with polarization-resolved second harmonic generation microscopy." J. Biophotonics 10, 1171-1179 (2017).

[24] R. A. Chipman, "Polarimetry." In: Handbook of Optics, 2nd ed., McGraw-Hill, New York, USA (1995).

[25] H. H. Chi, H. M. Katzin, C. C. Teng, "Histopathology of keratoconus," Am. J. Ophthalmol. 42(6), 847-860 (1956).

\section{Introducción}

El colágeno es la proteína estructural más abundante del cuerpo humano y componente mayoritario de la piel, los huesos, la esclera o la córnea [1,2]. Aunque existen más de 20 tipos de colágeno [3], el más abundante corresponde al tipo-I, formado por fibrillas de 20 a $100 \mathrm{~nm}$ de diámetro que agrupadas dan lugar a fibras de escala micrométrica. La organización espacial del colágeno tipo-I puede verse afectada por desnaturalización (edad, daño térmico,...) [4] o por procesos patológicos [5]. 
Una de las técnicas más extendidas para visualizar estructuras de colágeno es la microscopía de segundo armónico (SHG, del inglés Second Harmonic Generation). Ésta es una técnica de imagen no lineal resultado de la absorción cuasi-simultánea de dos fotones infrarrojos por un material con organización no centrosimétrica, como es el caso de los tejidos formados por colágeno [6, 7]. La microscopía SHG se ha empleado para diferenciar entre tejidos corneales sanos y patológicos [8] o para evaluar procesos quirúrgicos [9]. La señal SHG del colágeno es sensible a la polarización incidente $[10,11]$ y la modulación viene caracterizada por la hiperpolarizabilidad molecular del propio colágeno, la cual está relacionada con el ángulo de ensamblaje de la triple hélice molecular y con la orientación de las fibrillas [12,13].

El objetivo principal de la Tesis Doctoral ha sido el desarrollo de métodos matemáticos y técnicas polarimétricas para el análisis de desórdenes estructurales del colágeno que podrían estar relacionadas con ciertas enfermedades, lo que potencialmente permitiría un diagnóstico precoz. Las aportaciones originales de este trabajo son las siguientes:

- Desarrollo de un método matemático basado en el tensor de estructura para la obtención de parámetros de isotropía que permitan caracterizar la distribución espacial del colágeno a escala externa o micrométrica a partir de imágenes SHG [14].

- Construcción de un modelo teórico para analizar la sensibilidad a la polarización de la señal SHG en colágeno tipo I, permitiendo obtener una expresión analítica para el cálculo de la organización interna o a escala molecular [15]. La comprobación experimental permitió obtener por primera vez la relación entre la organización interna y externa del colágeno [16]. La sensibilidad a la polarización fue propuesta como método de diagnóstico clínico de la patología corneal conocida como queratocono.

- Aplicación del formalismo Stokes-Mueller para obtener tanto las propiedades de polarización del colágeno relacionadas con su estructura quiral [17], como para la optimización de la visualización local de las estructuras observadas en la imagen SHG.

\section{Métodos}

\subsection{Microscopio multifotónico polarimétrico}

Para el desarrollo del trabajo se ha empleado un microscopio multifotónico desarrollado en el Laboratoio de Óptica de la Universidad de Murcia [18]. El sistema combina un microscopio comercial invertido y un láser pulsado (Mira900f; Coherent, St Clara, CA) con $76 \mathrm{MHz}$ de frecuencia de repetición, 120 fs de duración de pulso y una longitud de onda infrarroja $(\lambda=800 \mathrm{~nm})$. El haz de luz atraviesa una unidad de escaneo compuesta por dos espejos galvanómetricos no resonantes que permiten escanear la muestra en el plano XY. Los espejos del escáner están conjugados con la pupila de entrada del objetivo del microscopio (20x, $\mathrm{NA}=0.5$ ) mediante dos sistemas telescópicos. Para registrar imágenes a diferentes profundidades se emplea un motor paso a paso acoplado al objetivo. La señal SHG se recoge en reflexión a través del objetivo y se aísla mediante un filtro espectral antes de llegar al tubo fotomultiplicador usado como sistema de registro. Para separar el camino de iluminación del sistema de detección, se emplea un espejo dicroico (ED) que refleja el haz de entrada y transmite la señal SHG. Un esquema del microscopio se muestra en la Fig. 1.

Para modular el estado de polarización de la luz incidente, se emplea un generador de estados de polarización (GEP) como indica la Fig. 1. Esta unidad fue diseñada para 3 configuraciones diferentes. Las dos primeras generan estados de polarización lineales y elípticos, respectivamente. Una descripción detallada puede consultarse en [15]. La combinación secuencial del polarizador lineal y la $\lambda / 2$ genera estados de polarización lineales. Al añadir la $\lambda / 4$ se generan estado elípticos (incluidos los circulares levógiro y dextrógiro). Tanto los lineales como los elípticos se obtienen cada $15^{\circ}$ de giro del eje rápido de la lámina. La tercera configuración (polarizador lineal y $\lambda / 4$ ) permite obtener 4 estados independientes de polarización orientando el eje rápido del retardador a $-45^{\circ}, 0^{\circ}, 30^{\circ}$ y $60^{\circ}$ [19]. Los 4 estados generados permiten a su vez calcular la primera fila de la matriz de Mueller de la muestra analizada [20], como se describe en detalle en la sección 2.4. 


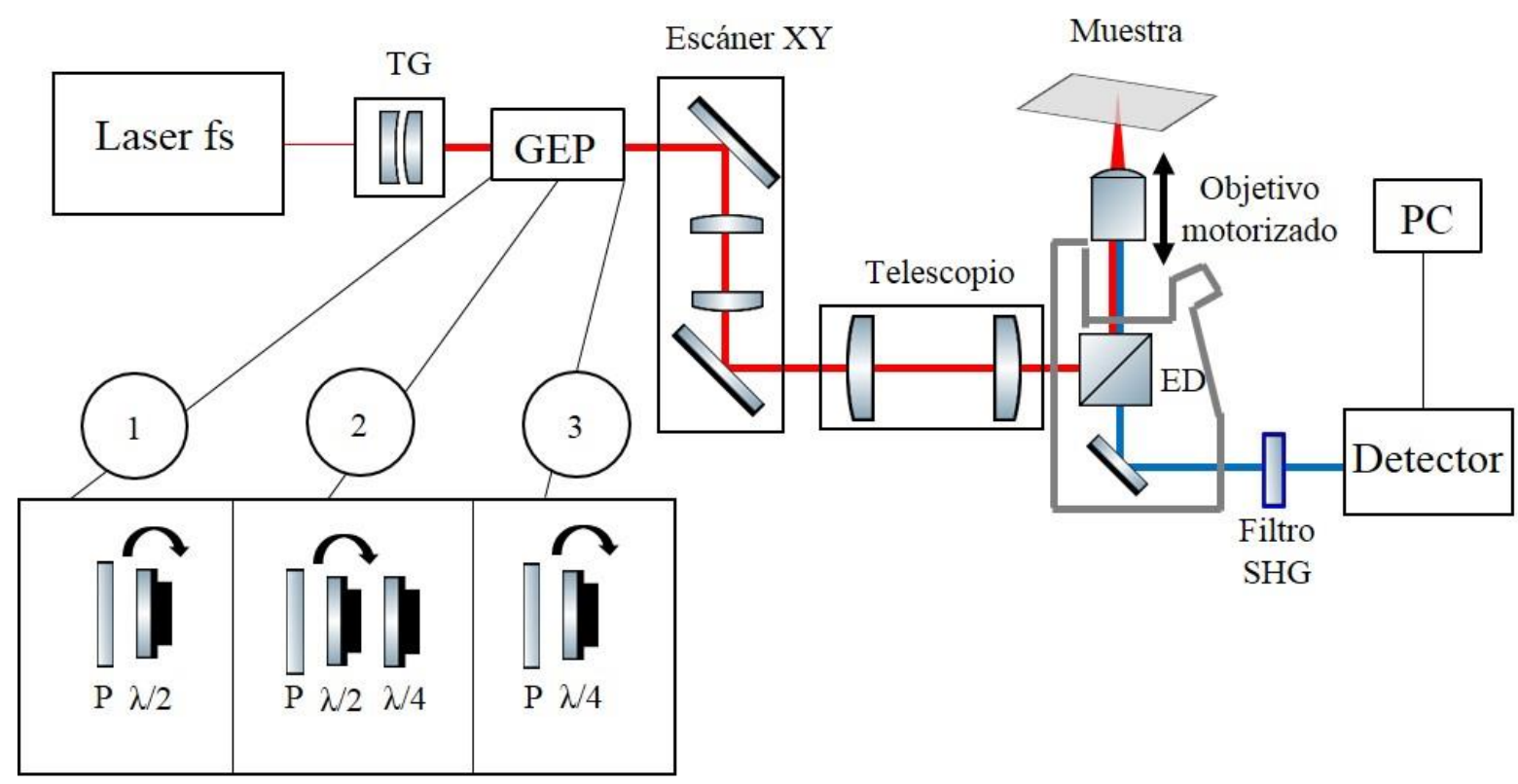

Fig.1. Esquema del sistema experimental empleado para la adquisición de imágenes SHG polarimétricas. TG: telescopio de Galileo; GEP: generador de estados de polarización; P: polarizador lineal; $\lambda / 2$ : lámina retardadora de media onda; $\lambda / 4$ : lámina retardadora de cuarto de onda; ED: espejo dicroico; PC: ordenador de procesado.

En este estudio se han analizado muestras de tejidos oculares formados por colágeno fibrilar tipo I, como son la córnea y la esclera. En particular se emplearon muestras tanto humanas como de varias especies animales. En particular aquí se mostrarán resultados en tejidos ex vivo de conejo (procedentes de la Facultad de Medicina de la Universidad de Valladolid) y humanos (donados por el Servicio de Oftalmología del Hospital Universitario Virgen de la Arrixaca de Murcia). Las muestras humanas se conservadas en Optisol ${ }^{\mathrm{TM}}$ y las animales en solución Medium 199 combinada con L-glutamina.

Para cada muestra, se registraron series de imágenes SHG con cada configuración del GEP, 12 para cada estado de polarización en las configuraciones 1 y 2, y 4 imágenes para la configuración 3. Cada imagen usada es el promedio de 3 imágenes individuales lo que permite aumentar la relación señal-ruido. El registro de imagen corresponde a un tamaño de 250 x 250 píxeles, para una resolución de $0.84 \mu \mathrm{m} /$ pixel y tiempo de adquisición de $\sim 1$ segundo. La potencia media empleada en el plano de la muestra varió entre 30 y 50 $\mathrm{mW} / \mathrm{cm}^{2}$, dependiendo del tipo de tejido. El sistema de microscopía se controla mediante un software propio desarrollado en LabVIEW ${ }^{\mathrm{TM}}$. El post-procesado de imagen se llevó a cabo mediante rutinas desarrolladas en MATLAB TM.

\subsection{Cálculo de la organización externa del colágeno}

Para caracterizar de forma objetiva el orden estructural de las muestras de colágeno se ha desarrollado una herramienta matemática basado en el tensor de estructura [21]. En particular se obtienen parámetros cuantitativos como el grado de isotropía o el grado de organización (GdO), la dispersión estructural o la orientación preferencial (OP) si la hubiere [14]. De forma resumida, el método computa, pixel a pixel, las derivadas direccionales a lo largo de los ejes cartesianos, para obtener los auto-valores asociados y con ellos la orientación y el nivel de organización de la estructura analizada. En la Fig. 2 se muestra un ejemplo de aplicación del tensor de estructura a una imagen generada por ordenador. Cuanto mayor sea la diferencia entre auto-valores $\left(\lambda_{\max } \mathrm{y} \lambda_{\min }\right)$ mayor es la organización de la estructura a lo largo de una dirección determinada. A partir de estos auto-valores espacialmente resueltos, se obtienen los mapas de GdO y OP. La desviación estándar del mapa de OP se ha definido como la dispersión estructural (DS).

Se ha establecido una escala numérica para diferenciar entre muestras alineadas (Gd0 $\geq 0.70$; DS $\leqslant 20^{\circ}$ ), parcialmente organizadas $\left(0.20 \leqslant \mathrm{GdO}<0.70 ; 20^{\circ}<\mathrm{DS} \leqslant 40^{\circ}\right)$ y desorganizadas $\left(\mathrm{GdO}<0.20 ; \mathrm{DS}>40^{\circ}\right)$ [14]. En los casos extremos, una muestra totalmente alineada tendrá un valor de $\mathrm{GdO}=1$ y una distribución espacial aleatoria presentará un $\mathrm{GdO}=0$. Por otra parte, la muestra o distribución de colágeno presentará una orientación preferencial cuando el histograma de OP pueda ser ajustado mediante una función gaussiana, tanto más organizada cuanto más estrecha y alta sea la campana de ajuste. 

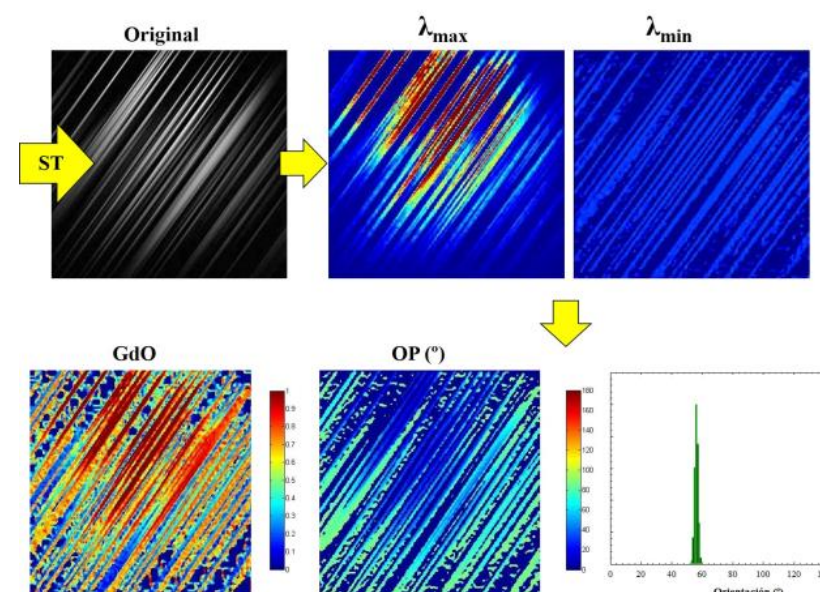
OP $\left({ }^{\circ}\right)$

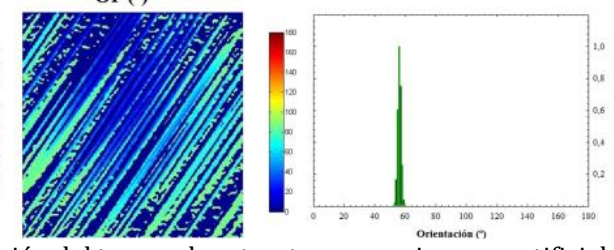

(2) $8 . \%$

Fig.2. Ejemplo de aplicación del tensor de estructura a una imagen artificial.

\subsection{Polarización y señal SHG: Organización molecular del colágeno}

Supongamos una fuente de luz láser polarizada (acimut $\chi$ y elipticidad $\psi$ ) con frecuencia fundamental $\omega$, incidiendo sobre un conjunto de $\mathrm{N}$ fibras de colágeno (con $\alpha_{\mathrm{j}}$ como ángulo de orientación de cada fibra con respecto a eje $\boldsymbol{X}$ ) con una dispersión estructural $D S$. La intensidad SHG en dirección backward viene dada por la expresión [15]:

$$
\tilde{I}_{2 \omega}=\sum_{j=1}^{N} I_{2 \omega, j}^{\alpha_{j}} \approx A(\theta, \varphi) \cdot B\left(\lambda_{\omega}, N A\right) \cdot C(\chi, \psi, \rho, D S)
$$

Por tanto, la intensidad de SHG depende de la dirección de radiación $(\theta, \varphi)$, la longitud de onda de la fuente de iluminación $\left(\lambda_{\omega}\right)$, la apertura numérica del objetivo (NA), el estado de polarización incidente $(\chi, \psi)$, la razón de hiperpolarizabilidades $\rho$ (relacionada con la escala interna del colágeno) y la organización externa de las fibras, $D S$. Tanto la configuración de registro (dirección backward) como la resolución del sistema experimental no permiten la visualización de las fibrillas que componen las fibras de colágeno, y que están relacionadas con la escala interna o nanoscópica aquí mencionada. Sin embargo mediante el análisis de la señal SHG polarizada es posible extraer información a este nivel mediante el cálculo del parámetro $\rho$, definido como [15]:

$$
\rho^{2}=\mathrm{L}_{H} / \mathrm{LV}_{\mathrm{V}}
$$

donde $L_{H}$ y $L_{V}$ corresponden a las intensidades SHG registradas para los estados de polarización lineal horizontal y vertical, respectivamente (obtenidas usando la configuración 1 del sistema experimental). Este parámetro varía entre $-3 \leqslant \rho \leqslant+3$ [22] y su valor absoluto disminuye en casos de desestructuración interna [4]. Además, se ha reportado que variaciones en el signo de $\rho$ están asociados a una inversión en la polaridad de las fibrillas de colágeno [10].

\subsection{Polarimetría Stokes-Mueller en las imágenes SHG}

En el apartado anterior se ha mostrado la relación entre la organización interna del colágeno y el comportamiento de la señal SHG polarizada. Sin embargo, es importante explorar la respuesta de la señal SHG para cualquier estado de polarización incidente y no sólo para luz lineal, ya que el cálculo de la organización interna tiene en cuenta sólo estados lineales y puede dar lugar a estimaciones erróneas, como puede ocurrir en muestras muy desorganizadas [23].

Para ello se ha hecho uso del formalismo Stokes-Mueller y de la configuración 3 del GEP. En concreto, se ha calculado la primera fila de la matrix de Mueller $M_{D}$ [17], cuyos elementos $m_{01}{ }^{S H G}(\mathrm{i}=0,1,2,3)$ permiten analizar (reconstruir) el comportamiento de la señal SHG para cualquier estado de polarización incidente mediante el cálculo de las propiedades de diatenuación $(D)$ de la muestra. Es parámetro se define como:

$$
D=\frac{\sqrt{\left(m_{01}^{S H G}\right)^{2}+\left(m_{02}^{S H G}\right)^{2}+\left(m_{03}^{S H G}\right)^{2}}}{m_{00}^{S H G}}
$$


que toma valores entre 0 y 1 . Además, $D$ está relacionado con la sensibilidad a la polarización, es decir, cómo es la dependencia entre la intensidad saliente de un sistema óptico y el estado de polarización de la luz incidente [24].

Por otra parte, los elementos de la primera fila de la matriz de Mueller pueden usarse para analizar el efecto de la polarización sobre la señal SHG de forma global, permitiendo así reconstruir imágenes SHG para cualquier pareja de puntos $(\chi, \psi)$ sobre la esfera de Poincaré (incluso aunque no sean físicamente realizables con el sistema experimental del que se dispone). Esto permite optimizar las imágenes SHG maximizando una métrica pre-definida utilizando la expresión [20]:

$$
I_{S H G}=M_{D} \cdot S(\chi, \psi)=\left(\begin{array}{llll}
m_{00}^{S H G} & m_{01}^{S H G} & m_{02}^{S H G} & m_{03}^{S H G}
\end{array}\right) \cdot\left(\begin{array}{c}
1 \\
\cos (2 \chi) \cdot \cos (2 \psi) \\
\sin (2 \chi) \cdot \cos (2 \psi) \\
\sin (2 \psi)
\end{array}\right)
$$

\section{Resultados}

\subsection{Organización externa}

En la Fig. 3 se muestran imágenes SHG registradas con luz polarizada lineal de muestras de esclera de bovino (muestra \#1, (a)), córnea humana control o sana (muestra \#2, (b)) y córnea humana patológica afectada por queratocono (muestra \#3, (c)). La información contenida en estas imágenes SHG es puramente estructural, con resolución a escala microscópica suficiente para mostrar evidencia de la distribución espacial del colágeno. Para cuantificar esta organización de forma objetiva, en la Fig. 4 se muestran los histogramas de OP correspondientes a las muestras \#1 y \#2.

Como puede observarse, el histograma correspondiente a la muestra de esclera presenta una DS significativamente alta (45을, asociada a la ausencia de OP y por tanto a una muestra desordenada. Por otra parte, el histograma relativo a córnea humana presenta un pico centrado en -5o con una dispersión estructural de 15ํㅜ , que corresponde a una muestra ordenada. Para una comparación más directa, la parte derecha de la Fig. 4 muestra un mapa comparativo de OP y DS, donde se observa cómo la dispersión de valores detectados por el método es mucho mayor en la esclera de bovino que en la córnea humana control. Aunque una inspección visual permite estimar el "estado de salud" del colágeno, la aplicación del tensor de estructura permite cuantificar y clasificar la organización de la distribución de las fibras de colágeno.
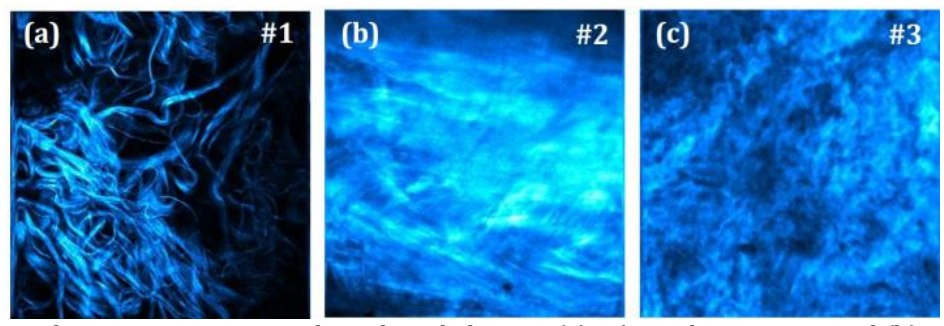

Fig.3. Imágenes SHG registradas para una muestra de esclera de bovino (a), córnea humana control (b) y córnea humana afectada por queratocono (c). Las imágenes tienen dimensiones de $210 \times 210 \mu \mathrm{m}^{2}$.
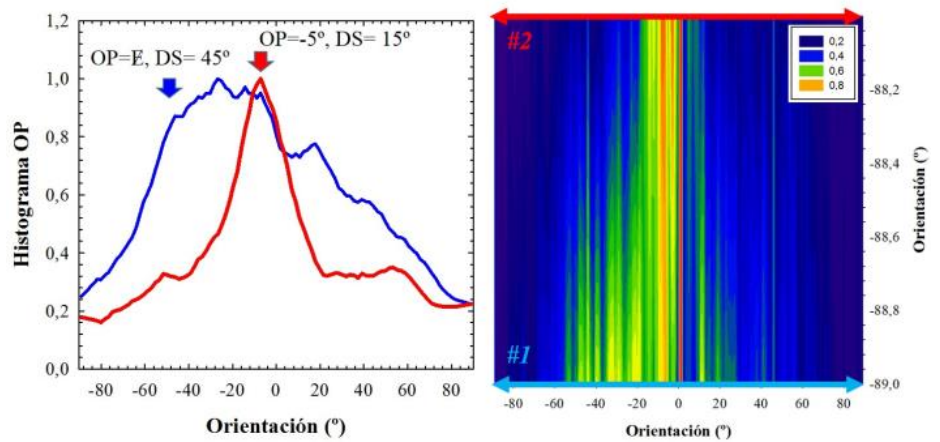

Fig. 4. Histogramas de OP correspondientes a las muestras \#1 y \#2 de la figura anterior (izquierda). Mapa comparativo de DS para ambas muestras (derecha) 


\subsection{Organización interna y señal SHG polarimética}

La Fig. 5 muestra las distribuciones de intensidad SHG en función del estado de polarización lineal incidente (imágenes registradas con la configuración 1 del GEP) para las muestras \# 1 y \#2. También se muestran los mapas de $\rho$ calculados a partir de la ecuación (2). La gráficas muestran que la modulación de la curva intensidad depende del estado estructural del colágeno: la dependencia con la polarización es tanto mayor cuanto mayor es la organización externa o el GdO. Además, la organización interna y la externa están relacionadas, de forma que cuanto mayor es el valor de $|\rho|$ mayor es GdO [17].

Por otra parte, el signo de $\rho$ determina la forma de la distribución de intensidad: para valores negativos aparece un máximo secundario en torno al estado de polarización vertical [16]. En las muestras aquí utilizadas el tejido de escleral presenta valor positivo de $\rho$, mientras que la córnea muestra una distribución de intensidad correspondiente a valores negativos de $\rho$.

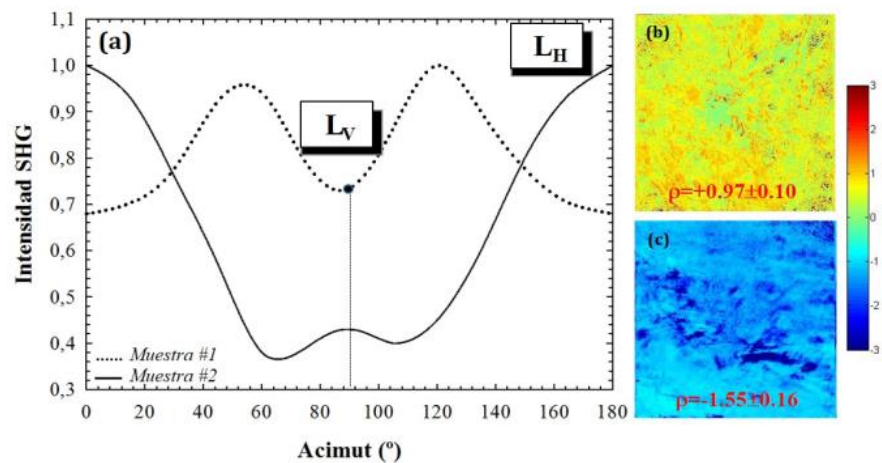

Fig. 5. Distribución de intensidad SHG en función del estado de polarización lineal incidente para las muestras \#1 y \#2 (izquierda). Mapas de $\rho$ para dichas muestras (derecha).

Por su parte las Fig. 6 (a) y 6(b) presentan los mapas de D espacialmente resueltos para las muestras \#1 y \#2, calculados haciendo uso de la ecuación 3. De nuevo se observa cómo la sensibilidad a la polarización crece con la organización externa. De hecho, la muestra \#2 presenta una mayor D y una menor DS que la muestra \#1. Sin embargo, lo interesante es comprobar si esta relación también se produce a escala interna o nanoscópica, ya que esta información no es visible en la imagen microscópica SHG y sólo puede extraerse modulando la señal SHG con polarización. Para ello la Fig. 6(c) representa los valores de $\rho$ frente a D para 19 muestras de tejidos oculares (incluyendo córnea y esclera) diferentes que pueden ser ajustados mediante la función dada en la figura con un ajuste $\mathrm{R}^{2}=0.93$. Estos resultados demuestran que la sensibilidad a la polarización de una muestra formada por colágeno también depende del estado de organización a escala nanoscópica.
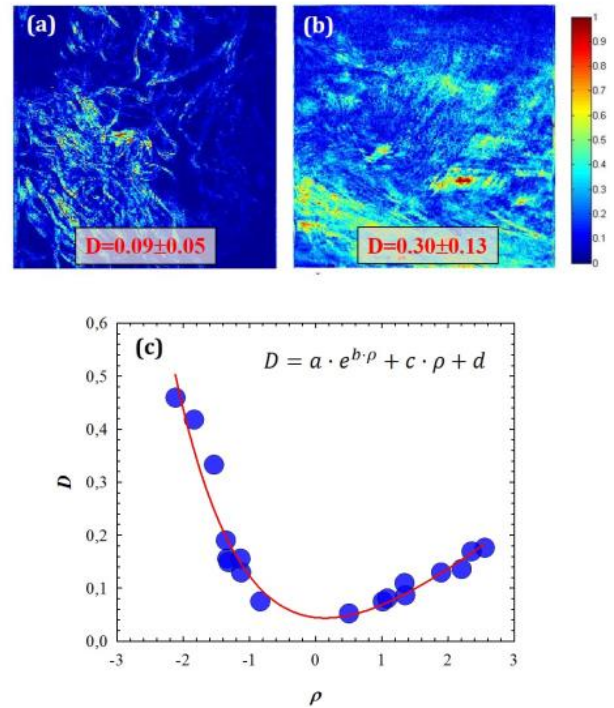

Fig.6. Mapas de D para las muestras \#a y \#b y valores de D frente a $\rho$ para 19 muestras de tejidos oculares diferentes. $(a=0.13, b=-$ $0.83, c=0.10, d=-0.09$ ). 


\subsection{Reconstrucción de imágenes SHG polarimétricas}

Tal como se ha comprobado en los apartados anteriores, la sensibilidad a la polarización depende de la organización del colágeno. Por tanto, es esperable que la mejora de la intensidad SHG o de la calidad óptica de imagen sea tanto mayor cuanto mayor sea dicha sensibilidad. En ese sentido, la Fig. 7 muestra imágenes SHG reconstruidas con máxima intensidad SHG y máxima relación señal-ruido (SNR) para las muestras \#1 y \#2. La mejora en intensidad y SNR reconstruidos corresponde a 17\% y 19\% para la muestra \#1, y $28 \%$ y $83 \%$ para la muestra \#2.
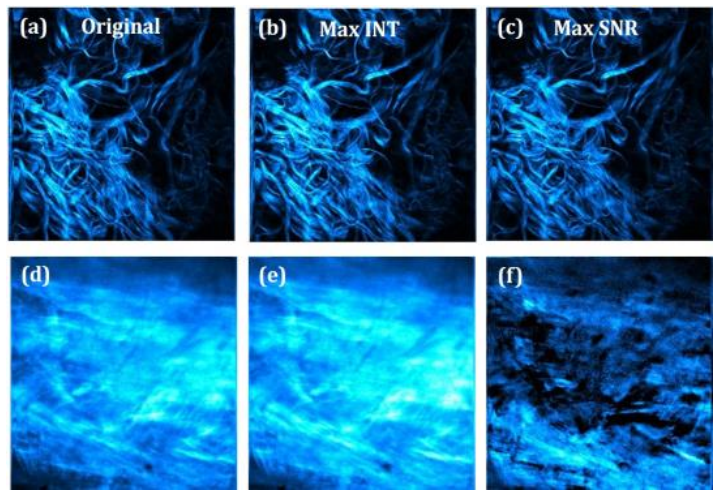

Fig.7. Imágenes SHG originales (muestras \#1 y \#2) y reconstruidas para máxima intensidad SHG (b, e) y máxima SNR (c, f).

La Fig. 8 representa los vectores de Stokes para los que se han obtenido las imágenes SHG con máxima intensidad y SNR. La máxima señal SHG se obtiene en aquellos estados lineales paralelos a la dirección preferencial de las fibras de colágeno. No obstante, en muestras no alinedas el estado de polarización que optimiza la señal puede corresponder a estados elípticos, como ocurre en el caso de la muestra \#1. Por otra parte, en términos de calidad óptica, los máximos valores SNR se obtienen para estados elípticos, lo que prueba que en algunos casos los estados óptimos para obtener imagen SHG no son los correspondientes a los estados lineales.

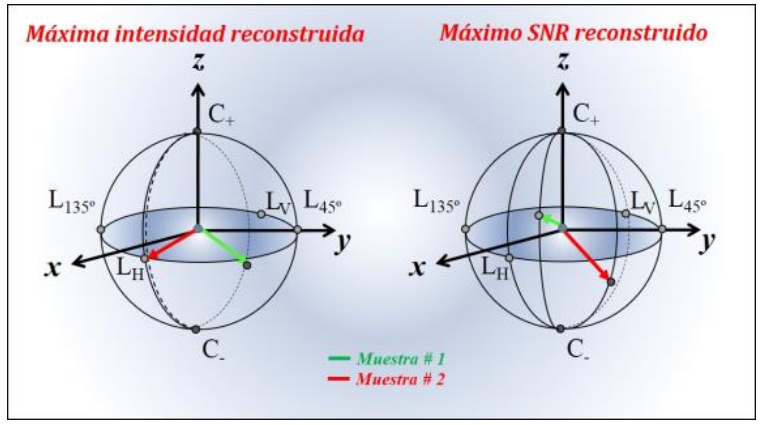

Fig.8. Vectores de Stokes representados sobre la esfera de Poincaré para los que se han reconstruido las imágenes SHG con máxi ma intensidad y SNR.

\subsection{Aplicación a un caso clínico: queratocono corneal}

Como se ha apuntado en la Introducción, determinadas patologías como el queratocono corneal causan desórdenes en el estroma corneal [25] que afectan gravemente a la biomecánica corneal, comprometen la visión e incluso requieren de un transplante corneal cuando están en estados avanzados. El problema para un diagnóstico temprano radica en la ausencia de signos clínicos en sus etapas iniciales. En este último apartado se propone la aplicación de las técnicas desarrolladas en esta Tesis Doctoral como método diferencial de diagnóstico precoz del queratocono corneal.

Si la patología produce un desorden local en el estroma corneal, la distribución regular de fibras de colágeno, como muestra la Fig. 3(c), perderá su orientación preferencial natural (Fig. 3(b)). La Fig. 9 compara los histogramas de OP en una córnea sana y otra afectada de queratocono, correspondientes a las muestras \#2 y \#3 de la Fig. 3. Como puede observarse, la muestra sana presenta una distribución con un pico centrado en -5ํㅜ (Fig. 9(a)) y una DS de 15ㅇ. Esta organización desaparece completamente en la córnea afectada, presentando una distribución homogénea en el histograma de PO (Fig. 9(b)). Para comprobar además el estado de salud a escala interna, se han obtenido las distribuciones de intensidad en función del estado de polarización incidente (empleando la configuración 1 del GEP) que se muestran en la Fig. 9(c). La 
distribución correspondiente a la córnea control (línea continua) muestra una modulación de señal mucho más acusada que la muestra patológica (línea discontinua), causando una disminución en el parámetro $\rho$ desde $-1.55 \pm 0.16$ hasta $-0.50 \pm 0.03$. Estos resultados indican una reducción acusada de la organización nanoscópica del colágeno debido a la desnaturalización del colágeno, lo que es coherente con las simulaciones de Chang y colaboradores [Chang et al., 2010].
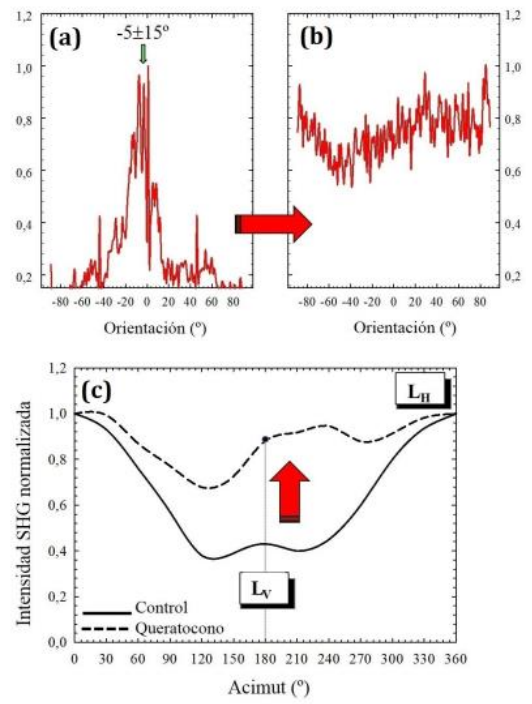

Fig.9. Distribuciones de PO (a, b) correspondientes a las muestras sana y afectada (a, b), respectivamente. Curvas de intensidad SHG en función del estado de polarización incidente lineal (c).

\section{Conclusiones}

En este artículo de revisión de Tesis Doctoral se han presentado las principales aportaciones del trabajo original al campo de la microscopía de imagen multifotónica y sus aplicaciones biomédicas. Se ha propuesto un método matemático basado en el tensor de estructura capaz de proporcionar información cuantitativa de la organización del colágeno a partir de imágenes SHG que permiten clasificar las muestras desde el punto de vista microscópico.

Por otra parte, se ha desarrollado un modelo teórico para analizar la dependencia de la señal SHG con la polarización incidente, lo que ha llevado a una expresión analítica para el cálculo de la organización interna o nanoscópica de las fibrillas que componen las fibras de colágeno, y no visibles mediante técnicas de microscopía convencional. Para la comprobación experimental, se ha modificado un microscopio multifotónico [18] incorporando un GEP con una triple configuración.

Se ha encontrado que la modulación de la señal polarizada SHG depende del grado de organización del colágeno, a escalas tanto externa como interna, y que además, la organización externa o micrométrica depende de la organización interna del colágeno. Es decir, los desórdenes en las distribuciones de colágeno visibles con microscopía multifotónica, tienen su origen a una escala más interna no visible con esta técnica.

Se ha utilizado el formalismo de Stokes-Mueller para analizar las propiedades del colágeno asociadas a la sensibilidad a la polarización. Los resultados han mostrado que dicha sensibilidad depende de la organización nanoscópica, y que ésta condiciona la organización externa. Finalmente, se ha empleado dicho formalismo para reconstruir imágenes SHG optimizadas que permitieran una mejor visualización de detalles en los tejidos formados por colágeno. Aunque en este trabajo se han aplicado los métodos al caso del queratocono corneal, éstos podrían usarse empleados en el diagnóstico clínico de cualquier patología que afecte al tejido conjuntivo, como son los casos de cáncer o neoplasia.

\section{Agradecimientos}

Financiado por Secretaría de Estado de Investigación, Desarrollo e Innovación (SEIDI), España (FIS201676163-R). 